\title{
The ARCS radial collimator
}

\author{
M.B. Stone ${ }^{1, a}$, J.L. Niedziela ${ }^{2}$, M.A. Overbay ${ }^{2}$ and D.L. Abernathy ${ }^{1}$ \\ ${ }^{1}$ Quantum Condensed Matter Science Division, Oak Ridge National Laboratory, Oak Ridge, Tennessee 37831, USA \\ ${ }^{2}$ Instrument and Source Division, Oak Ridge National Laboratory, Oak Ridge, Tennessee 37831, USA
}

\begin{abstract}
We have designed, installed, and commissioned a scattered beam radial collimator for use at the ARCS Wide Angular Range Chopper Spectrometer at the Spallation Neutron Source. The collimator has been designed to work effectively for thermal and epithermal neutrons and with a range of sample environments. Other design considerations include the accommodation of working within a high vacuum environment and having the ability to quickly install and remove the collimator from the scattered beam. We present here characterization of the collimator's performance and methodologies for its effective use.
\end{abstract}

Direct geometry time-of-flight neutron spectrometers often make use of large detector arrays to improve measurement efficiency [1]. Radial collimators in the scattered beam of such instruments have been found to effectively reduce the background scattering due to sample environments such as cryostats or furnaces [2]. Such collimators are often used in instrumentation with cold or thermal wavelength neutrons [3-7]. We discuss here the design and performance of a radial collimator for the Wide Angular Range Chopper Spectrometer (ARCS) at the Spallation Neutron Source [8]. ARCS is a high flux chopper spectrometer that operates with thermal to epithermal wavelength neutrons [9].

Figure 1 illustrates the location and orientation of the radial collimator. The ARCS instrument has two vacuum chambers separated by a large gate valve to facilitate sample environment changes without venting the larger detector vacuum chamber. To accommodate the out-ofplane detector coverage of the instrument, the height of the collimating blades or septa is $622 \mathrm{~mm}$. The inner radius of the collimator is $r_{1}=308 \mathrm{~mm}$, and the outer radius is $r_{2}=$ $462 \mathrm{~mm}$. The angular spacing between septa is $1.6^{\circ}$. The angular range of the collimator is $-30.5^{\circ}$ to $138.0^{\circ}$ with a $12.8^{\circ}$ wide portion in the forward scattering direction not collimated. This portion was not collimated to eliminate any scattering of the direct beam by the septa. The value of $r_{2}$ was chosen to be as large as possible and not interfere with the gate valve. The value of $r_{1}$ was chosen to be larger than the most often used sample environments at ARCS. The angular spacing was chosen to remove the background from scattering events outside of a $25 \mathrm{~mm}$ radius from the sample center. The collimator resides on a motorized lifting fixture. This allows the collimator to be either in the scattered beam, or stored below the sample environment when it is not needed. When in the operating position, the collimator oscillates with a full range of $1.6^{\circ}$ and a period of 21 seconds via a motorized Scotch yoke

a e-mail: stonemb@ornl.gov drive mechanism. Further details regarding the design are available in Ref. [8].

The septa are 12 micron thick Kapton foils coated on each side with 39 microns of enriched boron carbide $\left({ }^{10} \mathrm{~B}_{4} \mathrm{C}\right.$ with $\left.{ }^{10} \mathrm{~B}>96 \%\right)$ in an ultra-high vacuum compatible binder. The neutron absorption of ${ }^{10} \mathrm{~B}$ is large and resonance free over a broad range of wavelengths. The collimator blades represent an additional $22 \mathrm{~m}^{2}$ of surface area, adding a large vacuum load to the sample vessel. To assess the collimator impact on pumping speed, we examined 153 pump down cycles prior to the collimator installation, and 79 pump down cycles after installation. The pump down time is the time it takes to go from atmospheric pressure (between 700 and 720 Torr) to the point where one can open the ARCS gate valve (2e5 Torr). Figure 2 shows a histogram of pump down times with and without the collimator installed. The median of the distribution for the pump down time with the collimator versus without is higher, with actual values 32.4 and 17.9 minutes respectively. The mean values are shifted substantially from the median, registering 45.2 and 55.7 minutes without and with the collimator respectively. Although the increase in median pump down time does not substantially impact instrument operations, one must be mindful of the exposure of the collimator to atmosphere. Improvements in performance are achieved by taking care to vent the vessel with dry gasses and to minimize the time the collimator blades are exposed to atmosphere. The former of these is accomplished by using facility supplied dry gas with a dew point of -40 Celsius. The latter is achieved by good operational practices such as pumping down the sample chamber during long sample environment changes, or tightly capping the sample vessel when the vacuum is not active.

Figure 3 shows the measured scattering intensity as a function of neutron energy transfer $(\hbar \omega)$ and wavevector $(Q)$ transfer in a configuration without (a) and with (b) the radial collimator in place. This measurement was performed with the incident energy $E_{i}=60 \mathrm{meV}$ and the ARCS Fermi chopper and $T_{0}$ chopper operating 


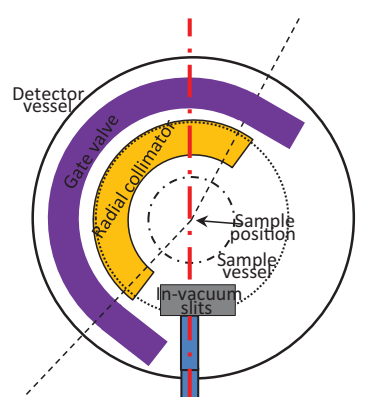

Figure 1. Plan view of the radial collimator in the ARCS sample vacuum chamber. The collimator is shown as orange and the gate valve between the sample and detector vacuum vessels is shown as purple. The dotted circle illustrates the maximum allowed size of sample environments at ARCS, radius $r=406.4 \mathrm{~mm}$. The dot-dashed circle represents the radius of sample environments typically used with the collimator, $r=203.2 \mathrm{~mm}$. The dashed lines emanating from the sample position illustrate the range of scattering angles for ARCS. The incident and transmitted neutron beam is shown as a red dot-dashed line.

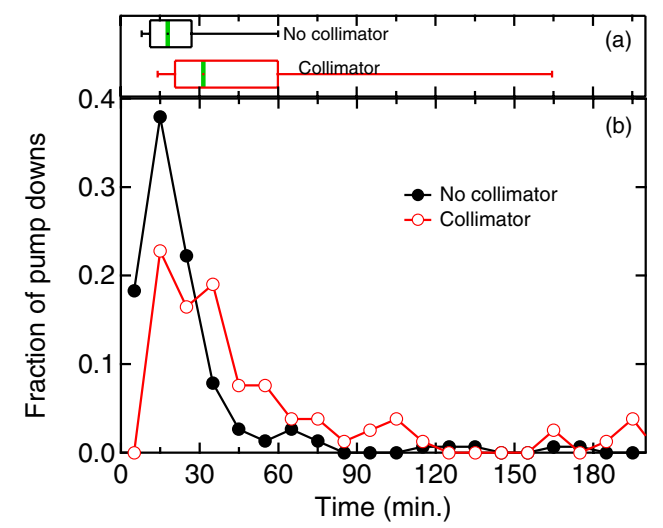

Figure 2. (a) A box and whisker plot illustrating the distribution in pump down times of the ARCS sample chamber. The green line is the median pump down time. The box range is between 25 and 75 percent and the whisker bottom and top are 10 and 90 percent respectively. (b) Distribution of pump down times for the radial collimator binned into 10 minute bins. Data points are shown at the center of the bins.

at 360 and $90 \mathrm{~Hz}$ respectively. No sample was at the sample position. The sample environment consisted of an aluminum cylinder with an inner radius of $72.8 \mathrm{~mm}$ and a wall thickness of $2 \mathrm{~mm}$, hereafter referred to as the aluminum thimble. In Fig. 3a, the scattering from the front and back of the sample environment manifests itself as a split elastic line at larger values of wave-vector transfer. There is also a great deal of inelastic signal due to the aluminum phonon-density of states. At small $Q$, there is some additional small angle scattering very likely due to air scattering from within the thimble. With the collimator in place, Fig. 3b, there is still a contribution from air scattering; however the elastic and inelastic signal are significantly reduced by the radial collimator.

We determined the gauge volume, the region from which scattering events are measured by the detectors, of the collimator by measuring the scattering intensity of a $6.35 \mathrm{~mm}$ diameter and $5 \mathrm{~cm}$ tall vanadium rod as a function of sample position. The rod was rotated about

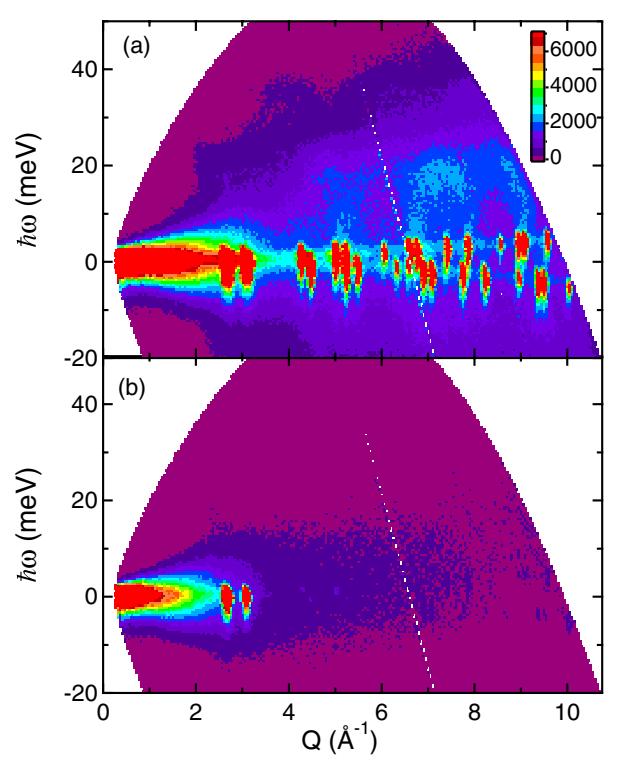

Figure 3. Scattering intensity as a function of energy and wavevector transfer for the empty aluminum thimble measured (a) without and (b) with the radial collimator in place. (a) shows the effect of a split elastic line at high momentum transfers, indicating the elastic scattering from the front and back of the sample environment, and additional scattering due to phonon scattering from the aluminum sample environment (b) shows that with the collimator installed, the split elastic line and the extra aluminum phonons are greatly reduced.

the vertical axis of the instrument and measured at many discreet angles. These measurements were performed with $E_{i}=75 \mathrm{meV}$ with the ARCS Fermi and $T_{0}$ choppers spinning at $420 \mathrm{~Hz}$ and $90 \mathrm{~Hz}$ respectively. Data were acquired both with and without the collimator installed while moving the vanadium rod through 99 positions in the vicinity of the nominal sample center position of the ARCS beamline, including a range of positions within $\pm 30 \mathrm{~mm}$ transverse to the neutron beam and $\pm 57 \mathrm{~mm}$ longitudinal to the neutron beam as shown in Fig. 4a. We transferred the scattering data to polar coordinates. Figure 4 shows the energy integrated dependent scattering intensity for several angular ranges. The effectiveness of the collimator increases with scattering angle to a maximum at $2 \theta=$ 90 degrees. This is a well understood effect and is fully characterized in Ref. [2].

The scattering volume measurements allow for a direct comparison to analytical descriptions of radial collimators as presented in Ref. [2], where Copley and Cook describe how a radial collimator influences a measurement using visibility functions. The visibility corresponds to an integration of the collimator transmission function over a thin annular sample at a given radius. The calculated visibility based upon the as-built collimator parameters and a total beam width of $50 \mathrm{~mm}$ is shown as a function of scattering angle and radius from the sample center position in Fig. 5c. Our calculation does not account for the missing blades in the forward direction of the asbuilt collimator. Determining the visibility based upon a large number of measurements of very thin vanadium cylinders would be expensive and require a significant 


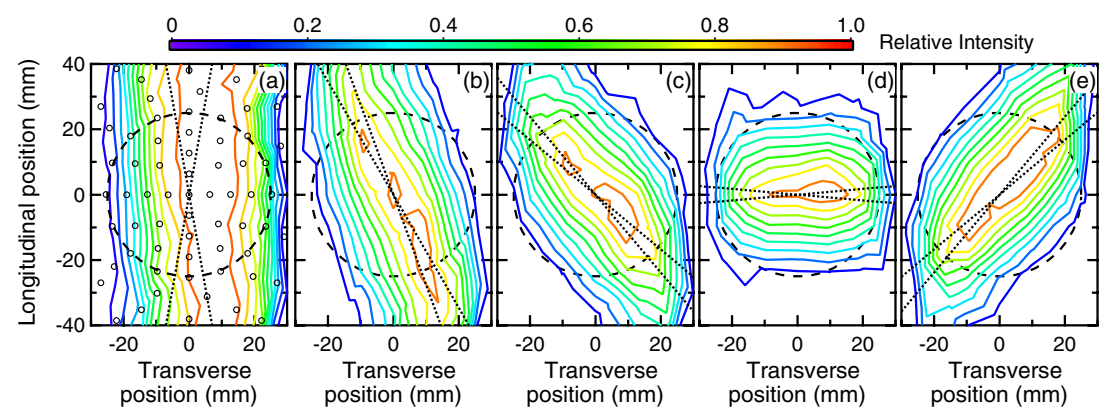

Figure 4. Relative scattering intensity of a $6.35 \mathrm{~mm}$ diameter vanadium rod as a function of sample position as viewed from above the sample position at the ARCS beam line. The origin of these figures is the beam line sample center position. The incident neutron beam travels from the bottom to the top of the figures. The dashed circle corresponds to a diameter of $50 \mathrm{~mm}$, the maximum beam width at ARCS. The contour plots are the measured scattering intensity integrated over all energy transfers but only integrating over a narrow range of scattering angle: (a) 0 to 10 degrees, (b) 20 to 30 degrees, (c) 40 to 50 degrees, (d) 85 to 95 degrees, and (e) 130 to 140 degrees. The dotted straight lines represent the range of scattering angle included in the integration. The integrated intensity has been normalized to the integrated intensity measured over the same range of scattering angle for the sample placed at the origin. The small circles in (a) are the positions of the vanadium rod used for the measurements.

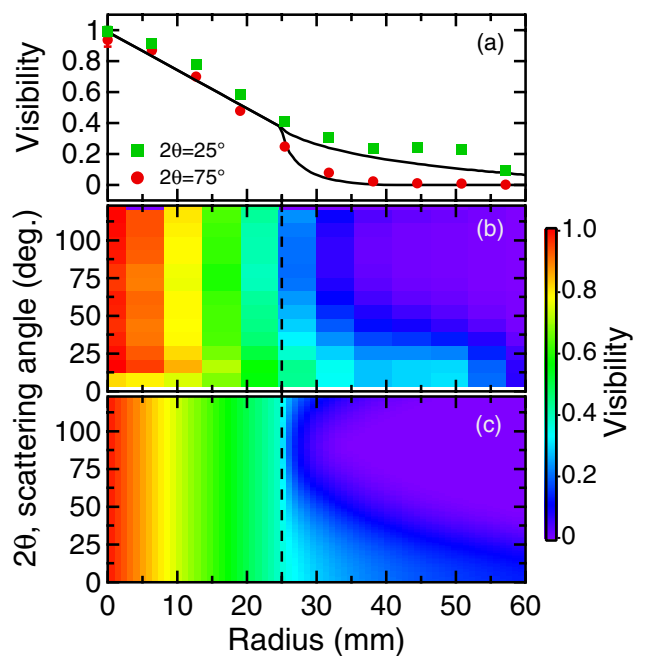

Figure 5. (b) Measured and (c) calculated visibility as a function of scattering angle and radius. The calculation corresponds to the as built parameters for the ARCS collimator. The dashed vertical line indicates the width of the incident beam used for the calculation. The measured visibility is based upon integrating the scattering intensity as a function of sample position for different scattering angles. The data integrated are the same as presented in Fig. 4. Panel (a) shows cuts through these data at fixed values of scattering angle integrated over a total width of 10 degrees.

amount of time to perform. Instead the measurements used for Fig. 4 were integrated to approximate the scattering due to a thin vanadium cylinder. The measurements were then binned in 10 degree increments of scattering angle and $6.35 \mathrm{~mm}$ steps in radii. The effective visibility was then normalized to the measured scattering intensity of the same vanadium rod placed at the sample center (radius $=0$ ) integrated over the same ranges of scattering angle. A final normalization to the maximum measured visibility of the entire dataset yields the data shown in Fig. 5b. For further comparison, we also plot cuts through the data and calculation at specific scattering angles in Fig. 5a. There is reasonable agreement between the measured approximate visibility and the calculated visibility, showing that the performance of the radial collimator is consistent with its specifications.

The absorption of the septa material decreases with increasing neutron energy. In order to quantify the effectiveness of the collimator as a function of neutron energy, we examined the scattering from a $0.152 \mathrm{~mm}$ thick and $5 \mathrm{~cm}$ tall high density polyethelene (HDPE) cylinder with a radius of approximately $42.5 \mathrm{~mm}$. Material placed at this radius is far out of the gauge volume of the collimator as demonstrated in Fig. 4. The HDPE cylinder was suspended at the sample position within a top-loading closed cycle refrigerator. The HDPE cylinder was measured at 23 different $E_{i}$ values between 30 and $5000 \mathrm{meV}$ with the collimator in and out of the beam. Three of these spectra are shown in Fig. 6. Recoil scattering of the carbon and hydrogen in the polyethelene are present for all spectra. Vibration modes can be seen clearly in Figs. 6a and b at smaller values of scattering angle. The recoil lines can easily be seen in Fig. 6c, where the hydrogen recoil is at a higher energy transfer than the carbon recoil scattering. The corresponding measurements shown in Figs. 6d-f show that the collimator is able to reduce the measured scattering intensity of the HDPE at all measured energies, but with decreasing efficiency at higher incident energies. We determine the energy dependent transmission from the ratio of the scattering intensities for measurements with and without the collimator in place. As the collimator is most effective near 90 degrees scattering angle, we integrate over scattering angles between 80 and 100 degrees. We plot the transmission in Fig. $6 \mathrm{~g}$ as a function of final neutron energy, $E_{f}$, where $\hbar \omega=E_{i}-E_{f}$. The series of measurements agree well with one another over more than three decades of neutron energy. Although there is no sharp cutoff in transmission, it becomes greater than 20 percent for final energies between 400 and $800 \mathrm{meV}$, which is greater than the transmission value calculated for an individual septa. Figure $6 \mathrm{~g}$ shows that the highest transmission rates obtained are 40 percent at the 

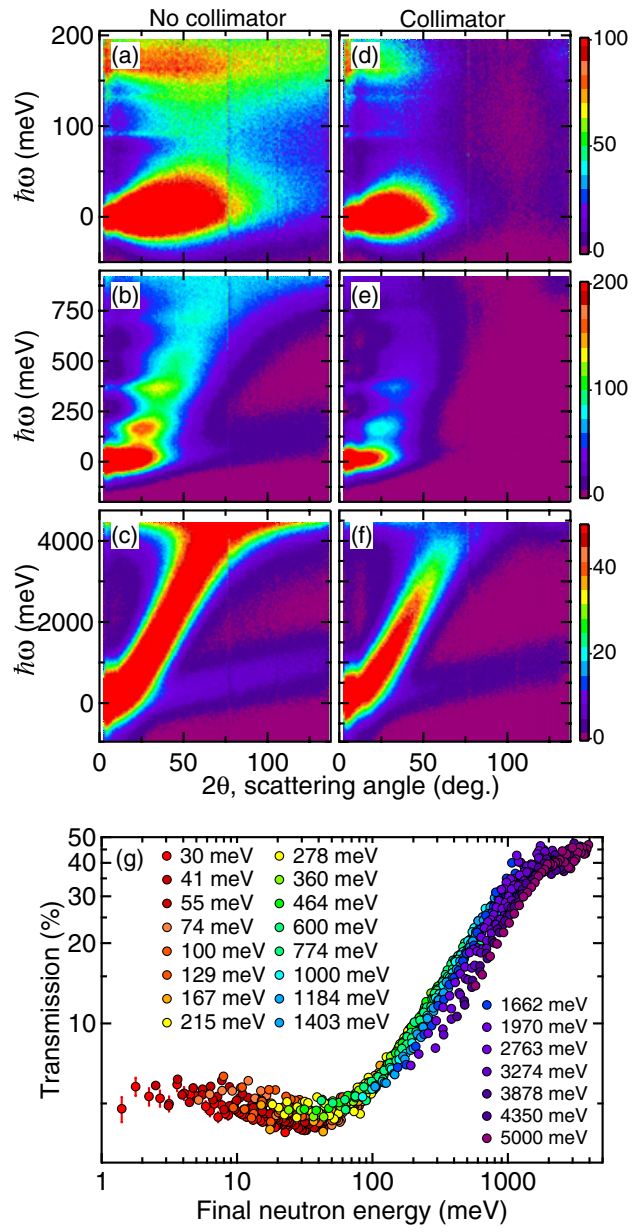

Figure 6. Measurements of HDPE cylinder over a series of incident energies to determine the energy dependent transmission of the ARCS radial collimator. (a) and (d) Were measured with $E_{i}=215 \mathrm{meV}$. (b) and (e) Were measured with $E_{i}=1000 \mathrm{meV}$. (c) and (f) Were measured with $E_{i}=5000 \mathrm{meV}$. (a)-(c) and (d)-(f) Were measured without and with the radial collimator in place. Data are plotted as the scattering intensity as a function of energy transfer and scattering angle. The intensity scale is the same for data measured with the same $E_{i}$ and is shown with the respective color bar on the right of the panels. (g) Neutron energy dependent transmission (percentage) for the ARCS radial collimator. Values in the legend correspond to the $E_{i}$ used for the measurement. Transmission values were determined by taking the ratio of the scattering intensity with and without the radial collimator in place for scattering angles between 80 and 100 degrees. The horizontal axis of panel $(\mathrm{g})$ is the final neutron energy, not the energy transfer.

largest energy transfers. These data demonstrate that the collective neutron absorption of the collimator works well over a range of neutron energies.
We have designed, built, installed, and operated a scattered beam radial collimator for the ARCS spectrometer at the SNS. The collimator works for both thermal and epithermal neutrons. Through a comparison to calculated visibility functions, we found that the collimator is operating effectively over a wide range of instrument conditions. We find that the use of this collimator significantly reduces the background scattering due to complicated sample environments, allowing for more time to be spent measuring samples rather than background characterizations. The collimator has been in use with the user program at the SNS since August of 2013, and has been used with both cryogenic and furnace sample environments with success.

We gratefully acknowledge the contributions of the support groups within the Neutron Sciences Directorate at ORNL including the Instrument Data Acquisition and Controls group, the Instrumentation Projects and Development group, the Vacuum Systems group, the Mechanical Shop, the Instrument Development and Target Support group, and the Instrument Support group. We thank JJ-Xray for their final design and production of the radial collimator. We thank Keller Technology Corporation for their manufacturing of the elevator and oscillator parts. The research at Oak Ridge National Laboratory's Spallation Neutron Source was sponsored by the Scientific User Facilities Division, Office of Basic Energy Sciences, U. S. Department of Energy.

\section{References}

[1] M. B. Stone, J. L. Niedziela, D. L. Abernathy, L. DeBeer-Schmitt, G. Ehlers, O. Garlea, G. E. Granroth, M. Graves-Brook, A. I. Kolesnikov, A. Podlesnyak, B. Winn, Rev. Sci. Instrum. 85, 045113 (2014)

[2] J. R. D. Copley and J. C. Cook, Nucl. Inst. and Meth. in Phys. Res. A 345, 313 (1994)

[3] J. R. D. Copley and J. C. Cook, Chemical Physics 292, 477 (2003)

[4] G. Ehlers, A. A. Podlesnyak, J. L. Niedziela, E. B. Iverson, and P. E. Sokol, Rev. Sci. Instrum. 82, 085108 (2011)

[5] D. Yu, R. Mole, T. Noakes, S. Kennedy, and R. Robinson, J. Phys. Soc. Jpn. 82, SA027 (2013)

[6] J. Mesot, S. Janssen, L. Holitzner and R. Hempelmann, J. Neutron Research, 3, 293 (1996)

[7] J. Ollivier and H. Mutka, J. Phys. Soc. Jpn. 80, SB003 (2011)

[8] M. B. Stone, J. L. Niedziela, M. J. Loguillo, M. A. Overbay, and D. L. Abernathy. Rev. Sci. Instrum., 85, 085101 (2014)

[9] D. L. Abernathy, M. B. Stone, M. J. Loguillo, M. S. Lucas, O. Delaire, X. Tang, J. Y. Y. Lin, and B. Fultz, Rev. Sci. Instrum. 83, 015114 (2012) 